\title{
Descripción de un evento de anidación del búho real (Bubo virginianus nacurutu Vieillot, 1817) y del búho moteado (Ciccaba virgata virgata Cassin, 1849) en Huila, Colombia
}

\section{Describing a nesting event of great horned owl (Bubo virginianus nacurutu Vieillot, 1817) and mottled owl (Ciccaba virgata virgata Cassin, 1849) in Huila, Colombia}

Orlando Padilla*

*Autor de correspondencia: orlandopadillarivera@gmail.com

Recibido: 03 de septiembre del 2018

Aceptado: 6 de diciembre de 2018

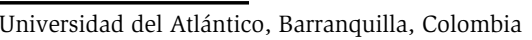

\begin{abstract}
Resumen
Palabras clave:

comportamiento reproductivo; nido; polluelos; Strigidae

En el presente estudio se registra por primera vez en Colombia un evento de anidación en especies de búhos Bubo virginianus nacurutu y Ciccaba virgata virgata en el departamento de Huila. Se describen aspectos como la época de anidación, tiempos de incubación de los eventos y la permanencia en sus nidos. Los resultados mostraron que $B$. virginianus nacurutu presentó un tiempo de incubación de 33 días, mientras que $C$. virgata virgata fue de 30 días. Respecto al tiempo de permanencia, se pudo evidenciar que la salida del polluelo de los organismos pertenecientes a C. virgata virgata ocurrió de 39-43 días a partir de la eclosión, en cambio, en C. virgata virgata se observó a los 50 días. La temporada de anidación documentada para ambas especies comprendió entre julio y noviembre de 2016, presentándose en una temporalidad más tardía a lo registrado en otros estudios en el continente americano.
\end{abstract}

Key words: breeding behavior; nest; chick; Strigidae

\section{Abstract}

The present study registers for the first time in Colombia a nesting event in two owl species, Bubo virginianus nacurutu and Ciccaba virgata virgata in the department of Huila, I describe aspects such as nesting season, incubation time and premanence in their nests. The results showed that $B$. virginianus nacurutu presented an incubation time of 33 days, while $C$. virgata virgata showed a time of 30 days. Regarding the permanence time on the nest it was possible to demonstrate that the exit of C.virgata virgata chick occurred 39-43 days after hatching and in B. virginianus nacurutu ocurred at 50 days. The nesting season for both species included the months of July and November 2016, developing later in time than recorded in other studies in the Americas. 


\section{Introducción}

A pesar de que las especies de búhos y lechuzas se encuentran ampliamente distribuidas en todo el mundo, la mayoría se distribuye en áreas tropicales y aproximadamente un tercio se distribuye en la región neotropical (Rangel y Enríquez, 2015). El conocimiento de los Strigiformes en Colombia es sumamente limitado, por ello existe la necesidad de realizar estudios que permitan comprender aspectos básicos como la variabilidad geográfica, estado poblacional, ecología reproductiva y comportamiento; ya que sin esta información es muy difícil conocer el estado actual, las tendencias poblacionales y los requerimientos ecológicos de los búhos (Chaparro-Herrera et al., 2015). El orden de los Strigiformes incluye la familia Strigidae que cuenta con 27 especies a nivel nacional; sin embargo, existe muy poca información sobre su biología reproductiva ya que pocos son los hallazgos y oportunidades de seguimiento (Chaparro-Herrera et al., 2015).

Particularmente, el búho real (Bubo virginianus nacurutu) es de amplia distribución que se encuentra en una gran variedad de hábitats, desde zonas de bosque, áreas abiertas, de crecimiento secundario y potreros. Es una especie estrictamente nocturna que se alimenta de diversas presas: pequeños mamíferos, aves, anfibios, reptiles, peces, insectos y otros invertebrados, así como conejos, ratones y aves acuáticas (Chaparro-Herrera et al., 2015). Se ha reportado que la época reproductiva es de diciembre a julio. Al respecto, es también escasa la información de individuos en condición reproductiva; se cuenta solamente con un reporte en el mes de enero en el bajo Cauca colombiano y con un reporte de volantón en abril en Guárico, Venezuela (Hilty y Brown, 2001). Generalmente utiliza nidos abandonados por otras aves grandes, en cavidades amplias de troncos o cerca del suelo en depresiones o en la base de árboles e incuban de uno a tres huevos (König et al., 2008; Holt et al., 2018a) durante 28-37 días (Chaparro-Herrera et al., 2015) y los juveniles vuelan a las 10 semanas (Holt et al., 2018a).

Por otro lado, del búho moteado (Ciccaba virgata virgata), solo existe información detallada sobre dieta, nidos y vocalizaciones de la zona de Petén en Guatemala (Gerhardt, 1991) y se reportan posturas de huevos entre febrero y mayo en Centroamérica (principalmente a finales de marzo en Guatemala) y entre septiembre y noviembre en el sur de su distribución, y juveniles entre febrero y marzo en el norte de Argentina (Holt et al., 2018a). Ponen de uno a tres huevos, tienen un periodo de incubación entre 28-30 días y los primeros vuelos del juvenil ocurren 9 entre los 27 y 33 días. Poco se conoce sobre su biología reproductiva en Colombia, excepto por reportes de aves en estado reproductivo que fueron encontradas entre febrero a mayo desde La Guajira hasta Córdoba, un polluelo en abril en Sierra Nevada de Santa Marta y un juvenil en julio en la región Caribe colombiana (Hilty y Brown, 2001). También se ha documentado que anidan en cavidades o ramas gruesas entre epífitas o en nidos abandonados de otras aves más grandes y ponen entre uno y tres huevos blancos (Gerhardt et al., 1994; König et al., 2008; Holt et al., 2018b). En este trabajo se describe un evento de anidación de Bubo virginianus nacurutu y de Ciccaba virgata virgata como contribución al escaso conocimiento de estas especies.

\section{Materiales y métodos}

\section{Área de estudio}

Realizando observaciones de aves en el sur del departamento del Huila, se detectaron dos nidos de las especies Bubo virginianus nacurutu - búho real y Ciccaba virgata virgata - búho moteado. La ubicación del nido de búho real fue a $3,6 \mathrm{~km}$ al sur del corregimiento de Río Loro del municipio de Gigante en una zona de escarpes rocosos con vegetación herbácea y cactáceas, a $50 \mathrm{~m}$ de la vía nacional entre Gigante y Garzón; mientras que el nido de búho moteado se encontró en el municipio del Agrado, en inmediaciones de zonas de pastoreo en la vereda Cabuyal dentro de un estrecho cañón rocoso con paredes verticales que sirve de curso a un arroyo (afluente del río Magdalena) rodeado de vegetación de rastrojo. Ambos nidos se encontraban alejados de centros poblados, a una altitud de $720 \mathrm{~m}$ y se hallaban encima de la cota de inundación del embalse el Quimbo. Toda la región comprendía originalmente ecosistemas de matorral xerófilo y bosque seco tropical (Pizano y García, 2014) (figura 1 a y b).

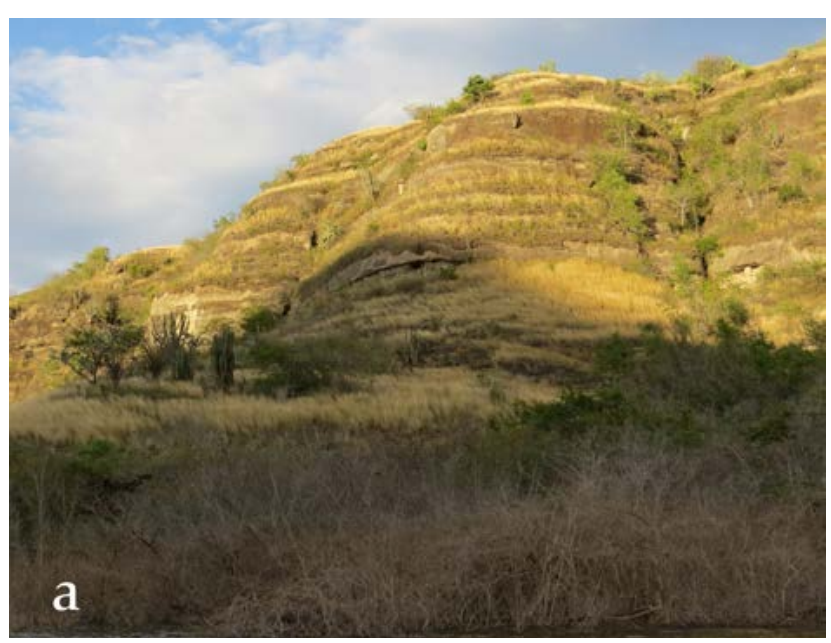




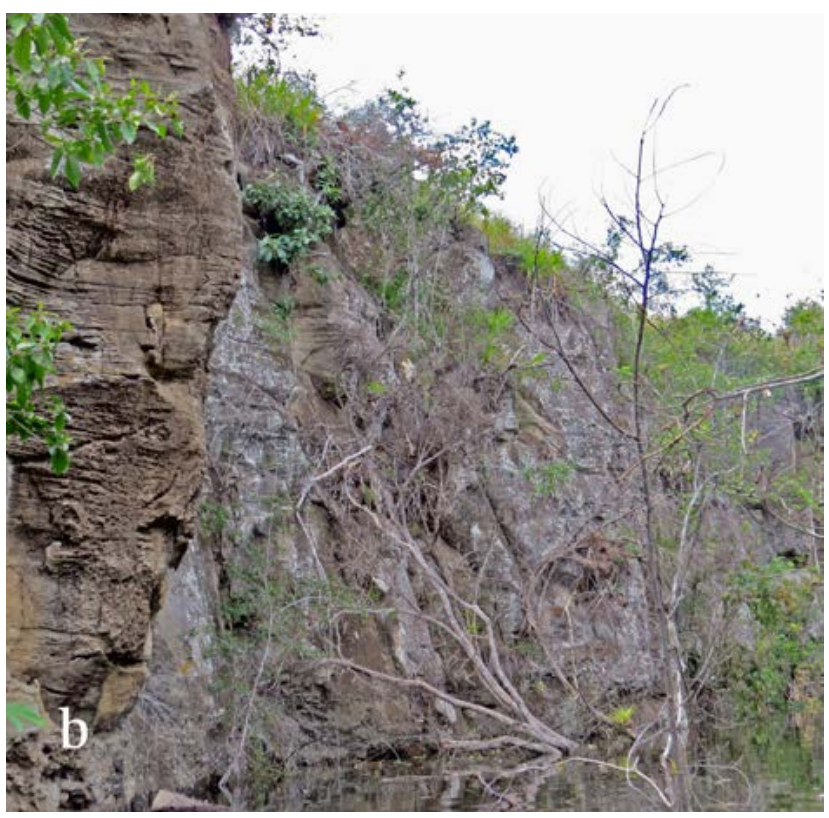

Figura 1. a. Aspecto de la localidad del nido de búho real y b. del nido de búho moteado.

Las observaciones de campo se realizaron entre agosto y octubre de 2016, durante la transición de época seca en agosto-septiembre a época lluviosa de octubrenoviembre. Para establecer el seguimiento de los nidos se registró la fecha, hora, presencia del parental, número de huevos, número de polluelos, fotografía y observaciones. Se evitó la manipulación del nido, de huevos y polluelos para garantizar la mínima intervención en el ciclo de cría.

Las visitas a los nidos de los búhos ( $\mathrm{n}=21$ para el búho real y $n=15$ para el búho moteado) siempre se realizaron durante las horas de luz entre las 6:00 y las 18:00 h.

\section{Resultados}

\section{Descripción de los nidos}

\section{Bubo virginianus nacurutu}

Estuvo constituido por trozos de ramas secas, era de forma ovalada y medía $80 \mathrm{~cm}$ de longitud. Se ubicaba a tres metros sobre el nivel del agua y debido a lo elaborado y el tamaño de la estructura del nido, se cree que se trataba de un nido abandonado por bandurrias (Theristicus caudatus (Boddaert, 1783)), especie que construye nidos similares que se han registrado en la misma zona (O. Padilla obs. pers.) (figura 2).

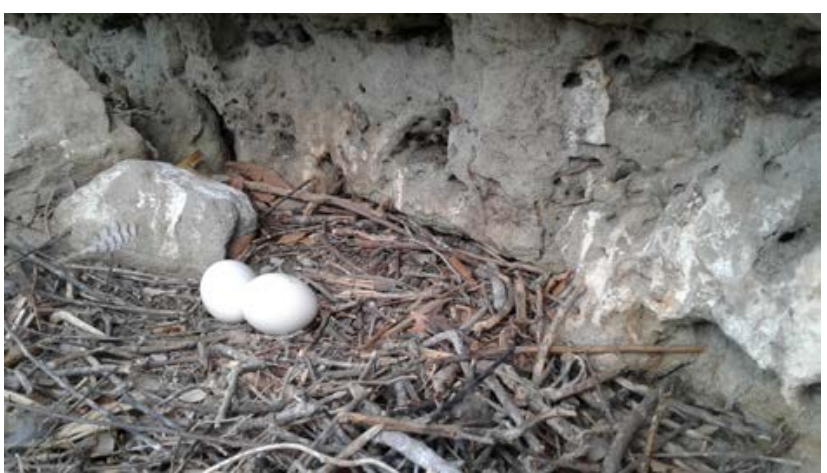

Figura 2. Aspecto del nido y evidencia de la postura de huevos.

\section{Ciccaba virgata virgata}

El nido de búho moteado no tenía material vegetal acumulado. Se ubicaba en una cavidad de $26 \mathrm{~cm}$ de diámetro, abierto en la parte superior y situado a cuatro metros de altura en un acantilado de tal manera que no se podía ver desde el suelo el contenido del mismo (figura 3).

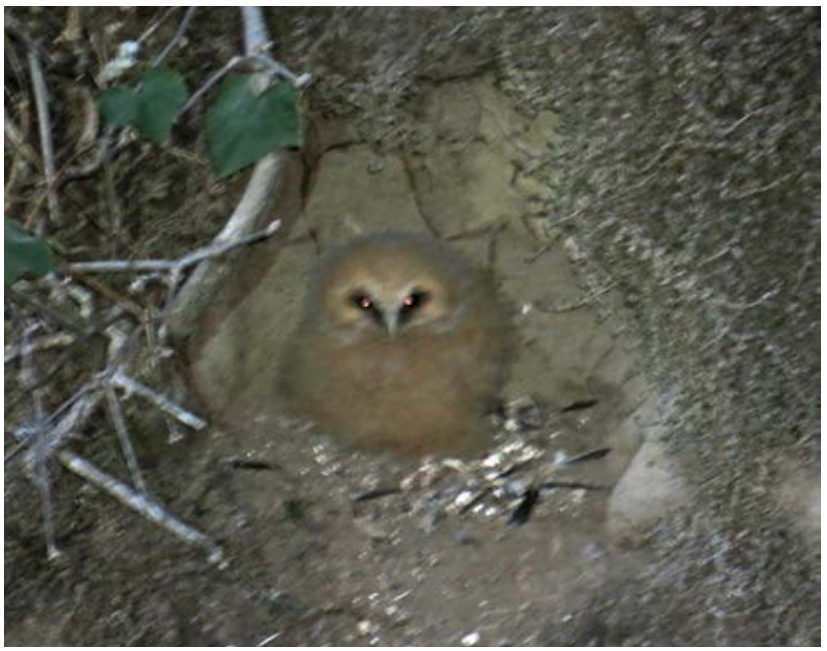

Figura 3. Aspecto del nido y polluelo.

\section{Observaciones en el seguimiento de los nidos}

\section{Bubo virginianus nacurutu}

El hallazgo del nido de búho real se produjo el 2 de agosto de 2016. Contenía dos huevos blancos y a partir de las fechas de eclosión de los polluelos (el primero, el 20 de agosto; el siguiente, entre el 23 y 25 de agosto) se calcula una fecha de postura de los huevos a mediados de julio de 2016, con un periodo de incubación aproximado de 33 días comprendido entre el 18 julio y 21 agosto. Al eclosionar 
los polluelos, presentaron su característico diente de huevo en el extremo de la ranfoteca, el cual desapareció entre los 33 y 37 días (entre el 25 y 29 de septiembre). Se registró la presencia de presas en el nido en dos ocasiones que correspondieron a plumaje de Crotophaga ani (Linnaeus, 1758) (Aves: Cuculiformes: Cuculidae) y dos Sigmodon hirsutus Burmeister, 1854 (Mammalia: Rodentia: Cricetidae) (figura 4).

Uno de los polluelos desapareció entre el 25 de agosto y el 5 de septiembre. El tiempo del polluelo sobreviviente en el nido fue de 52 días hasta su salida del área del nido (entre el 20 de agosto y 11 de octubre; 6,9 semanas de vida). En general, el ciclo de cría desde la puesta calculada hasta la salida del nido fue de 116 días (16,5 semanas) (figura 5).

Por otro lado, durante el $90 \%$ de las visitas $(\mathrm{n}=21)$ se observó cuidado parental cada vez que se aproximaba el observador al nido, pues el parental se posaba en una percha cercana a observar (figura 6).
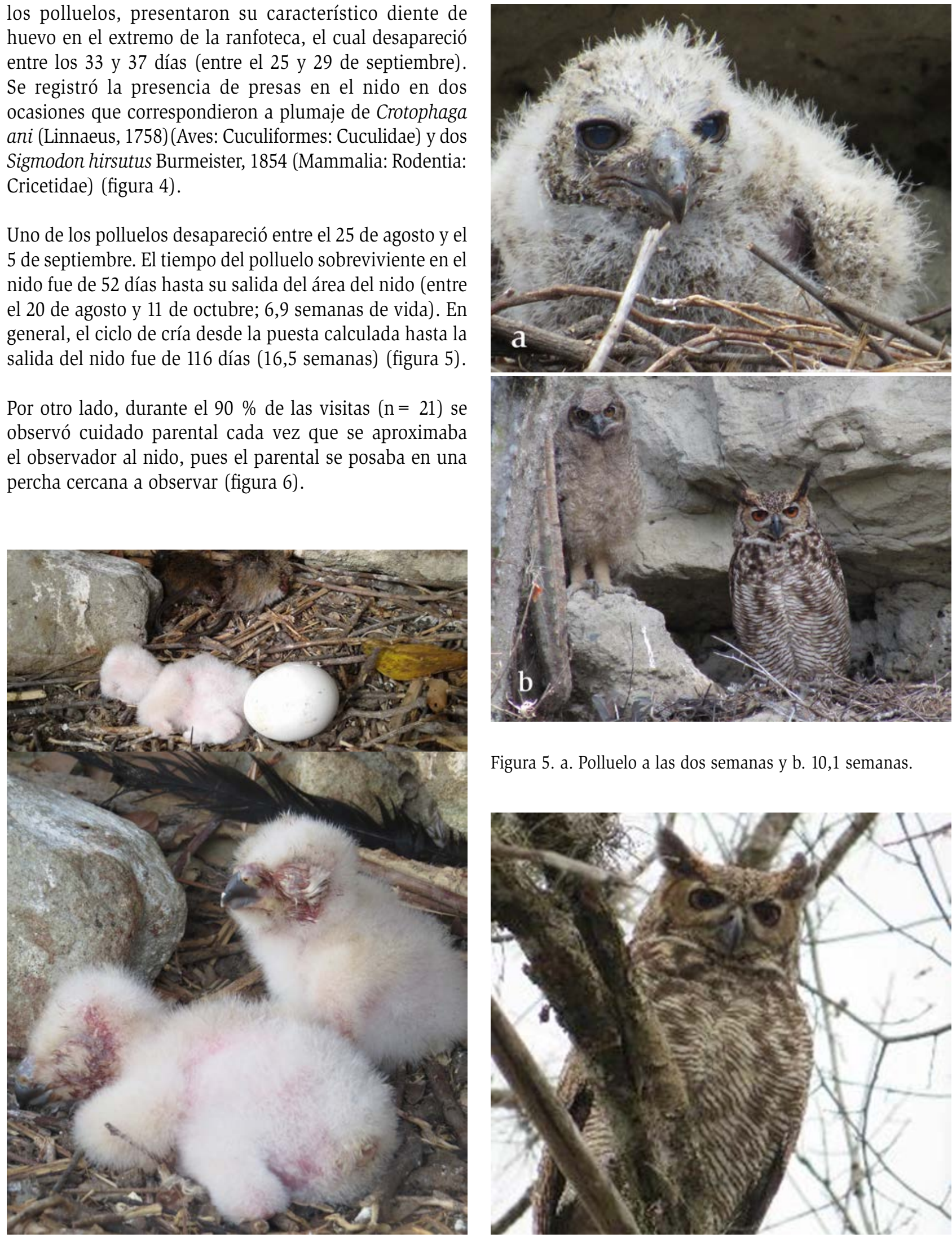

Figura 5. a. Polluelo a las dos semanas y b. 10,1 semanas.

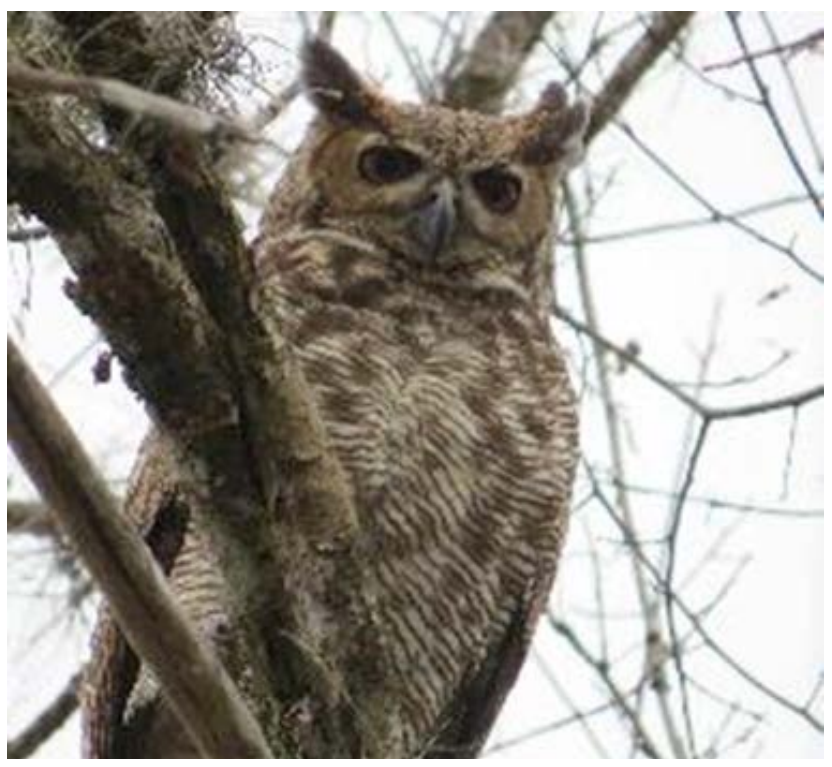

11 Figura 4. Evidencias de eclosión e ítems alimenticios.

Figura 6. Presencia del parental durante las observaciones. 


\section{Ciccaba virgata virgata}

El nido de búho moteado se encontró el 10 de agosto de 2016 y contenía un huevo que eclosionó entre el 16 y el 24 de agosto. Se estima que la postura del huevo ocurrió entre el 16 y el 24 de julio y que la salida del volantón debió ocurrir a los 30 días entre el 16 y 24 de septiembre; sin embargo, este último evento ocurrió el 3 de octubre, de 9 a 13 días más tarde.

En el ejemplar no se evidenció diente de huevo desde que se observó por primera vez. El día 21 de septiembre de 2016 se observó evidencia de alimentación de un ave pequeña no identificada alrededor del polluelo (figura 7).

En general, el ciclo de cría desde la incubación hasta la fuga del nido comprendió 76 días (10,8 semanas) (figura 8). En el $66 \%$ de las visitas al nido $(n=12)$ el parental estuvo presente y permaneció en la vegetación cercana al nido (figura 9). Por último, se visitó el área circundante con reproducción de llamado el 10 y el 15 de octubre, sin obtener registros ni respuesta a los llamados reproducidos.

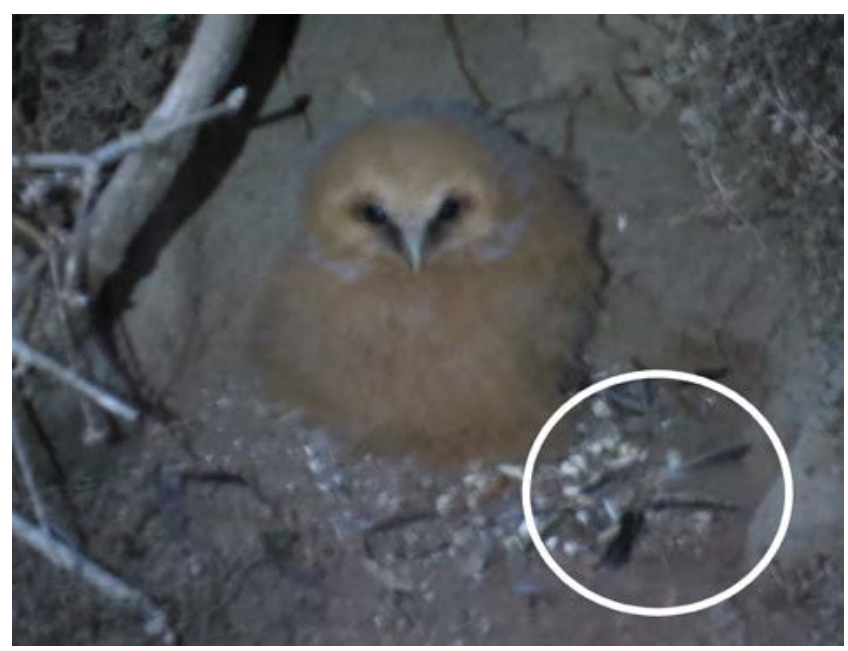

Figura 7. Evidencia del nido e ítem alimenticio. En el círculo se evidencian restos de un ave no identificada.
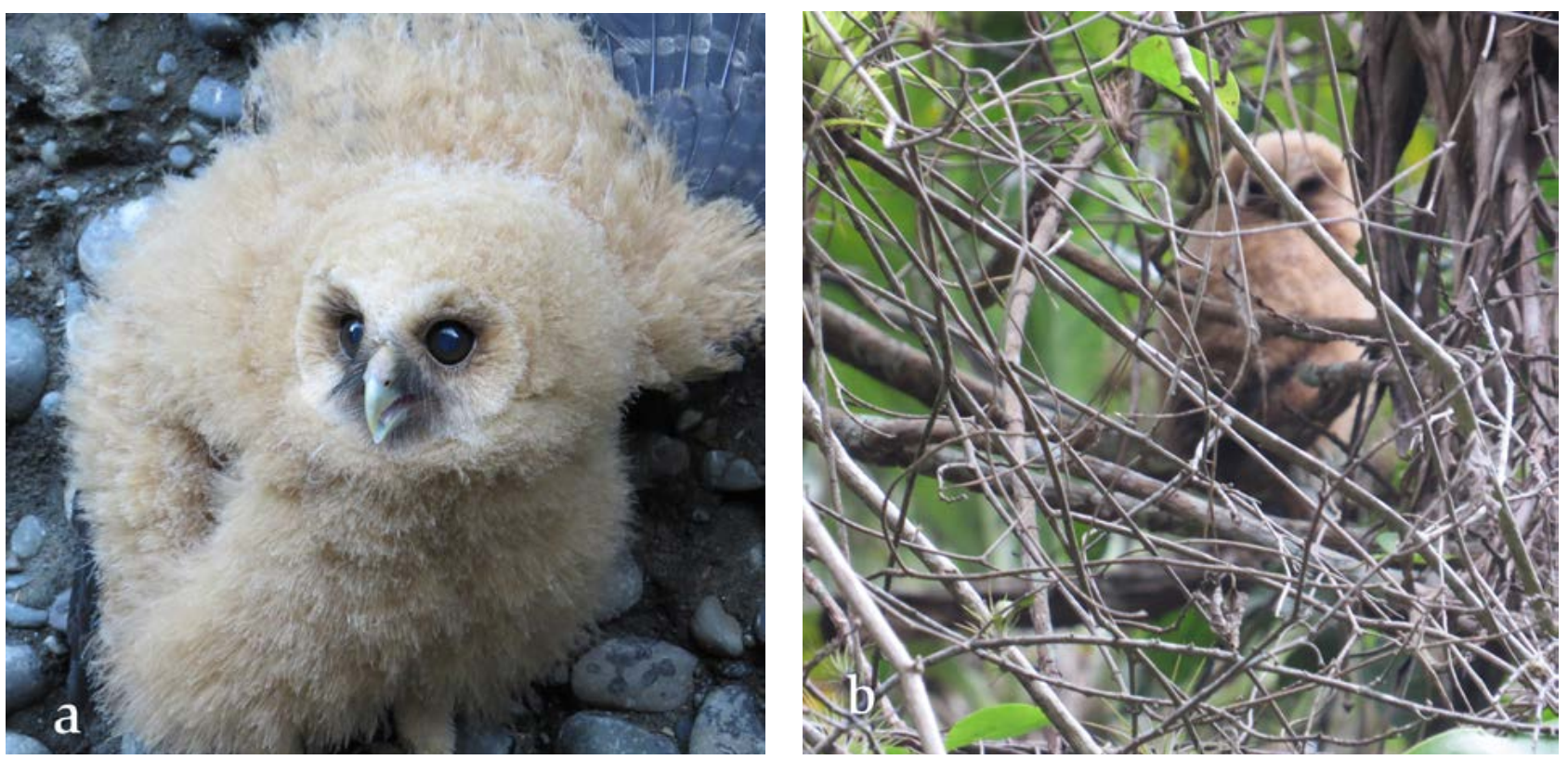

Figura 8. a. Volantón de Ciccaba virgata virgata fuera del nido (3 de octubre 2016); b. Volantón fuera del nido (4 de octubre 2016). 


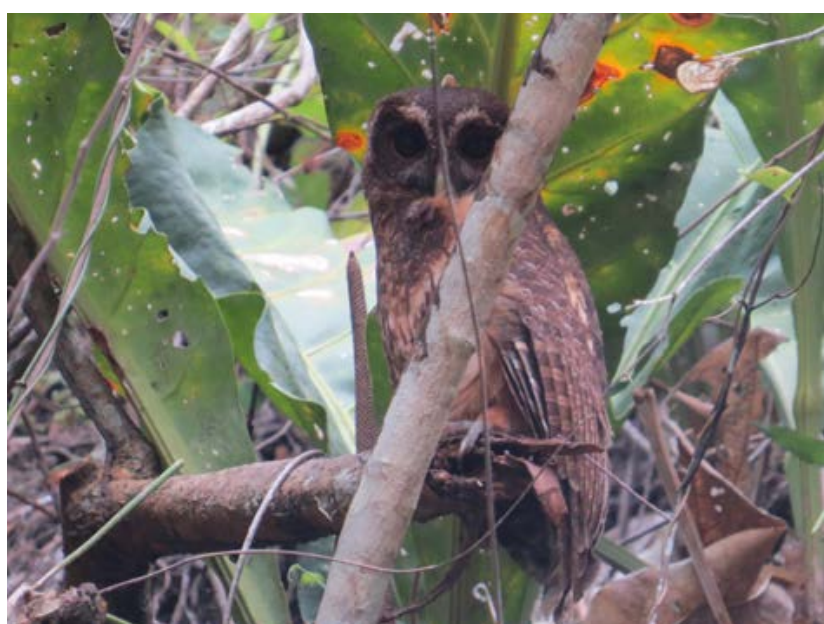

Figura 9. Presencia de parental de Ciccaba virgata virgata durante las observaciones.

\section{Discusión}

\section{Bubo virginianus nacurutu}

Según un registro en Canadá, los huevos de búho real son incubados por la hembra entre 30 y 37 días, y es alimentada por el macho durante este período (Stuart et al., 1998). En Colombia se ha reportado un periodo de incubación entre 28 y 35 días (Chaparro et al., 2015), rangos que corresponden a lo registrado en este trabajo. Después de la eclosión, los pichones permanecen en el nido entre seis y siete semanas (Stuart et al., 1998) y comienzan a volar en las semanas $10 \mathrm{y}$ 12 de edad (König y Weick, 2008). Este tiempo de abandono del área del nido corresponde con lo observado en la localidad de Gigante donde el polluelo se observó fuera del nido el 6 de octubre de 2016 a la edad de 6,5 semanas (figura 10) y lo abandonó el 11 de noviembre a las 12 semanas.

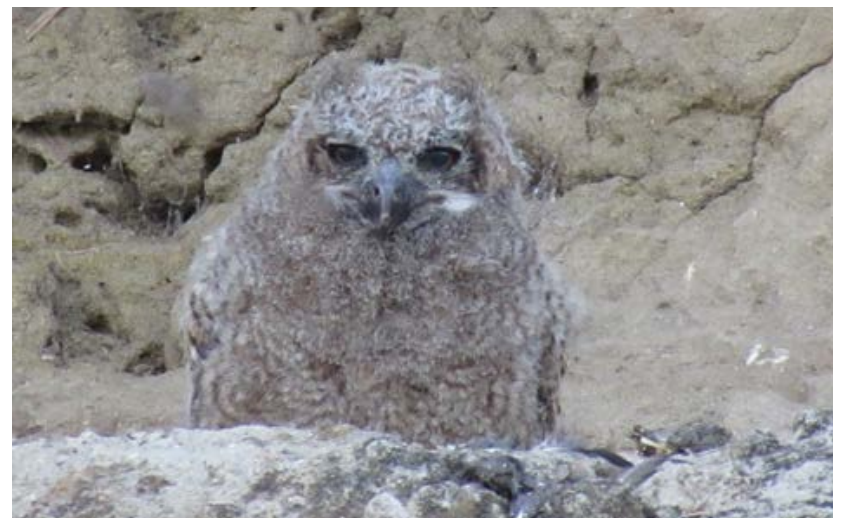

Figura 10. Evidencia salida de la estructura del nido a las 6,5 semanas (Fotografía J. Zúñiga).
En Norteamérica el inicio de la puesta se reportó más cerca a la mitad del año en la medida que aumenta la latitud y la altitud. En poblaciones norteñas ponen huevos más tarde que las sureñas; y en las de tierras altas, más tarde que las de tierras bajas (Holt y Drasen, 2001). En Colombia se han encontrado individuos en condición reproductiva en el mes de enero (Hilty y Brown, 2001); adicionalmente existen registros fotográficos de juveniles en los departamentos de Córdoba (5 de mayo de 2018), Bolívar (17 de febrero de 2018) y Antioquia (18 de octubre de 2017) (e-Bird, 2018). También, el 8 de agosto de 2015 se observó un volantón de aproximadamente 50 días en el departamento del Huila, cerca del municipio de Gigante (O. Padilla obs. pers.) En el departamento del Atlántico en área rural del municipio de Polonuevo se encontró un volantón el 21 de abril de 2017 en un nido sobre una palma amarga (Sabal mauritiiformis autor) (O. Padilla obs. pers.). En este reporte se evidencia la postura de huevos a mediados de julio y abandono del nido en noviembre, lo que permite inferir que estos búhos pueden reproducirse en cualquier época del año.

En otros países neotropicales y del Cono Sur se tienen reportes fotográficos de inmaduros y juveniles entre 2010 y 2018; por ejemplo, en México hay reportes de mayo y abril, en Honduras de mayo, en Brasil de julio, septiembre y octubre; Chile en noviembre y en Argentina en agosto y octubre (e-Bird, 2018) y en el norte de Venezuela se observaron 2 volantones en noviembre de 2014 (O. Padilla obs. pers). De acuerdo a lo anterior, la primera parte del año se perfila como época de cría en el hemisferio norte en contraste con el hemisferio sur, exceptuando el caso de Venezuela.

\section{Ciccaba virgata virgata}

El nido estuvo ocupado desde el inicio por un solo huevo y, según se conoce, el tamaño de la postura oscila entre uno o dos huevos y se cree que si pone un huevo o no pone ninguno podría ser por la escasez de alimento (Enríquez, 2006). En Colombia se han registrado polluelos en el mes de abril, individuos en condición reproductiva en febrero y mayo, y jóvenes en julio (Hilty y Brown, 2001). Similarmente ocurrió en México donde se han hallado nidos en mayo y junio (Enríquez, 2006). En Guatemala se han documentado posturas a finales de marzo y en Costa Rica un nido con dos huevos en febrero y sus respectivos polluelos en abril (Astorga, 2017). En el caso del nido monitoreado en este estudio, se encontró el polluelo entre 3 y 5 meses más tarde respecto a los anteriores referentes.

Otros registros fotográficos de volantones fuera de nido provienen de Centroamérica y Suramérica (Ecuador, 
Guatemala, Honduras, Costa Rica y México) en abril, mayo, junio y julio entre los años 2016 y 2018 (e-Bird, 2018), lo que confirma el primer semestre del año como temporada de cría. La incubación se reporta en un promedio de 30 días para Ciccaba nigrolineata autor (especie afín) (Pernis y Middleton, 1985). También se conocen tiempos de eclosión en Guatemala de 28 días para Ciccaba virgata (Gerhardt et al., 1994), lo que concuerda con los 30 días calculados en el nido observado.

Cuando el período de incubación ha finalizado, la hembra permanece cerca del nido mientras que el macho continúa alimentando a la hembra y a los polluelos (Enríquez, 2006). En este caso no se observó eventos de alimentación, en contraste, se observó escasa presencia de parentales (con $33 \%$ de las visitas con padres presentes después de alrededor de 16 días de vida del polluelo), lo que podría indicar que estos pueden dejar el nido simultáneamente para realizar otras actividades o vigilar desde perchas ocultas. Finalmente, los juveniles empiezan a volar aproximadamente al mes de nacidos, entre los 27 a 33 días de edad (Enríquez, 2006). En este caso el polluelo no se mostró dentro del rango esperado, ya que permaneció en el nido entre 9 y 13 días más.

\section{Conclusiones}

La fenología reproductiva de las especies estudiadas fueron eventos más tardíos con respecto a la información publicada sobre otras localidades de Colombia. El $B$. virginianus podría ser una especie con un amplio espectro temporal para la reproducción en Colombia y la $C$. virgata igualmente podría exhibir amplio espectro temporal. Además, el tiempo adicional de permanencia del polluelo en el nido de este último puede indicar alguna variante del comportamiento reproductivo por corroborar.

Finalmente, es importante destacar que es pertinente monitorear, describir y cuantificar otros eventos de anidación en diferentes regiones el país para comparar el comportamiento, desarrollo de polluelos y temporalidad del ciclo de cría de ambas especies.

\section{Referencias}

Astorga, J. 2017. Primer registro de nidificación sobre el suelo del Búho café (Ciccaba virgata), en Grecia, Costa Rica Huitzil. Revista Mexicana de Ornitología 18(2): 242-245.

Chaparro-H, S., Córdoba. S., López, J.P., Restrepo, J.S. y Cortés. O. 2015. Los Búhos de Colombia. En: Enríquez, P.,
Editor. Los Búhos Neotropicales: Diversidad y Conservación. ECOSUR, México D.F.

E-Bird. 2012. eBird: Una base de datos en línea para la abundancia y distribución de las aves [aplicación de internet]. eBird, Ithaca, New York. Disponible: http://www.ebird.org. Consultado: 14 de agosto de 2018.

Enríquez-Rocha, P. 2006. Ficha técnica de Ciccaba virgata. En: Escalante-Pliego, P., Editor. Fichas sobre las especies de Aves incluidas en el Proyecto de Norma Oficial Mexicana PROY-NOM-ECOL-2000. Parte 2. Instituto de Biología, Universidad Nacional Autónoma de México. Bases de datos SNIB-CONABIO Proyecto No. W042. México, D.F.

Gerhardt, R.P. 1991."Mottled Owls (Ciccaba virgata): Response to Calls, Breeding Biology, Home Range, and Food Habits. Boise State University Theses and Dissertations. http://scholarworks.boisestate.edu/td/736. Consultado: 14 de agosto de 2018.

Gerhardt, R., Bonilla, N., Mcannis, D. y Flattens, C.J. 1994. Breeding Biology and Home Range of two Ciccaba owls. Wilson Bulletin 106 (4): 629-639.

Hilty, S.L. y Brown, W.L. 2001. A Field Guide to the Birds of Colombia. Princeton University Press, New Jersey.

Holt, D. y Drasen, S. 2001. Early nesting by great horned owls in Montana. Journal of Raptor Research 35(1): 66-67.

Holt, D., Berkley, R., Deppe, C., Enríquez Rocha, P., Petersen, J., Rangel, L., Segars, K., Wood, K. y Marks, J. 2018a. Great Horned Owl (Bubo virginianus). En: Del Hoyo, J., Elliott, A., Sargatal, J., Christie, D.A. y De Juana, E., Editores. Handbook of the Birds of the World Alive. Lynx Edicions, Barcelona.

Holt, D., Berkley, R., Deppe, C., Enríquez Rocha, P., Petersen, J.L., Rangel Salazar, J.L., Segars, K., Wood, K., Kirwan, M., Marks, J. y Boesman, P. 2018b. Mottled Owl (Ciccaba virgata). En: Del Hoyo, J., Elliott, A., Sargatal, J., Christie, D.A. y De Juana, E. Editor. Handbook of the Birds of the World Alive. Lynx Edicions, Barcelona.

König, C. y Weick, F. 2008. Owls of the World. Christopher Helm Publishers, London.

Pernis, C. y Middleton, A. 1985. Owls. In enciclopedia of birds. Facst on file publications, New York.

Pizano, C. y García, H. 2014. El Bosque Seco Tropical en Colombia. Instituto de Investigación de Recursos Biológicos Alexander von Humboldt (IAvH). Bogotá, D.C. 
Rangel, J.L. y Enríquez, P. 2015. Aves en la región Neotropical. Stuart, C., Smith, G. y Rohner, C. 1998. Great horned owl En: Enríquez, P. Editor. Los Búhos Neotropicales: Diversidad (Bubo virginianus). En: Poole, A. Editor. The Birds of North y Conservación. ECOSUR, México. America Online. Cornell Lab of Ornithology, Ithaca.

Citar como: Padilla, O. 2019. Descripción de un evento de anidación del búho real (Bubo virginianus nacurutu Vieillot, 1817) y del búho moteado (Ciccaba virgata virgata Cassin, 1849) en Huila, Colombia.

Intropica 14(1): 8-15. DOI: https://doi.org/10.21676/23897864.2696 